$\checkmark$ Research Square
Preprints are preliminary reports that have not undergone peer review.

They should not be considered conclusive, used to inform clinical practice, or referenced by the media as validated information.

\title{
Osmotic stress activated protein kinase 5 (SAPK5) positively regulates immune response and blast resistance in rice
}

Xiaobo Zhu

Sichuan Agricultural University

Xiaoyu Long

Sichuan Agricultural University

WeiWei Ma

Sichuan Agricultural University

Hui Shi

Sichuan Agricultural University

Hong Yi

Sichuan Agricultural University

Li Xu

Sichuan Agricultural University

Wei Mao

Sichuan Agricultural University

Junjie Yin

Sichuan Agricultural University

Min He

Sichuan Agricultural University

Weitao Li

Sichuan Agricultural University

Li Song

Sichuan Agricultural University

Long Wang

Sichuan Agricultural University

Jiali Liu

Sichuan Agricultural University

Hai Qing

Sichuan Agricultural University

Yu Bi

Sichuan Agricultural University

Mingwu Li 
Sichuan Agricultural University

\section{Kun $\mathrm{Hu}$}

Sichuan Agricultural University

\section{Tuo Qi}

Sichuan Agricultural University

\section{QingQing Hou}

Sichuan Agricultural University

\section{Xuewei Chen}

Sichuan Agricultural University

Jing Wang ( $\sim$ jingwang406@sicau.edu.cn )

https://orcid.org/0000-0002-7984-1392

\section{Original article}

Keywords: SnRK2, protein kinase, phosphorylation, immune response, disease resistance, rice

Posted Date: August 11th, 2020

DOI: https://doi.org/10.21203/rs.3.rs-54599/v1

License: (1) (1) This work is licensed under a Creative Commons Attribution 4.0 International License. Read Full License 


\section{Abstract \\ Background}

The sucrose non-fermenting 1 (SNF1) related protein kinase 2 (SnRK2) family proteins are plant-specific Ser/Thr protein kinases that are mainly involved in response to abiotic stress. However, their regulation roles on immune response and disease resistance in crops are largely unknown.

\section{Results}

In this study, we report the characterization of a rice SnRK2 family member, osmotic stress activated protein kinases 5 (SAPK5), on plant immune response. The SAPK5 protein is localized in cytoplasm and possesses kinase activity. The kinase activity of SAPK5 is promoted upon treatment with chitin, a conserved pathogen-associated molecular pattern (PAMP). Knockout of SAPK5 compromises rice immune response and resistance against the fungal pathogen Magnaporthe oryzae.

\section{Conclusions}

Our study demonstrates that SAPK5 positively regulates immune response and disease resistance in rice and provides new insights into the regulatory roles of SnRK2 proteins on plant immunity.

\section{Background}

The sucrose non-fermenting 1 (SNF1) protein kinase family from plant belongs to a class of Ser/Ter protein kinase which is named as SNF1 in yeast, AMP activated protein kinase (AMPK) in mammals and SNF1-related protein kinase (SnRK) in plants and green algae (Mao et al. 2019). The proteins in this family are evolutionarily conserved and can be divided into three subfamilies, namely SnRK1, SnRK2 and SnRK3, based on sequence similarity and domain structure (Hrabak et al. 2003). Members from plant SnRK1 subfamily are orthologs of the SNF1/AMPK which mainly regulate cellular sugar/energy homeostasis (Martínez-Barajas and Coello 2020). The proteins form a complex by employing SnRK1a as the catalytic subunit, SnRK1 $\beta$ and SnRK1Y as the regulatory subunits to phosphorylate key enzymes of the related metabolic processes (Martínez-Barajas and Coello 2020). Members from plant SnRK3 subfamily are usually able to interact with calcineurin B-like proteins $(\mathrm{CBL})$ thus also termed as $\mathrm{CBL}$ interacting protein kinases (CIPK) (Yu et al. 2014). The CBL-CIPK/SnRK3 signaling pathway is a Ca ${ }^{2}$ dependent pathway that regulates response to both biotic and abiotic stimuli (Yu et al. 2014). Members from plant SnRK2 subfamily usually participate in response to various abiotic stresses. For examples, SnRK2.3 regulates drought response in Arabidopsis; SAPK8 and TaSnRK2.3 regulate freezing, drought and salt tolerance of rice and wheat respectively (Tian et al. 2013; Tan et al. 2018; Zhong et al. 2020). 
Members of SnRK2 subfamily can further be classed into three groups, including the group I of which the kinase activity is not activated by abscisic acid (ABA), the group II of which the kinase activity is not activated or activated weakly by ABA, and the group III of which the kinase activity is strongly activated by ABA (Mao et al. 2019). Ten members from SnRK2 subfamily have been identified in rice by homology searching the rice genome databases. They are designated as osmotic stress activated protein kinases (SAPKs) based on their characteristics reveled (Kobayashi et al. 2004). These ten kinases, i.e. SAPK1 through SAPK10, can be divided into three subclasses: subclass I contains SAPK4, 5, 6 and 7, subclass II consists of SAPK1, 2 and 3, and subclass III comprises SAPK8, 9 and 10 (Kobayashi et al. 2004; Kulik et al. 2011). All SAPK members are activated by hyperosmotic stress, and three of these proteins, SAPK8, SAPK9 and SAPK10, are activated by ABA (Kobayashi et al. 2004).

Protein kinases catalyze the phosphorylation of certain proteins by adding phosphate group(s) to substrate(s), whereby regulating various biological processes including plant immunity (Park et al. 2012; Meng and Zhang 2013). As a class of Ser/Thr protein kinases, SnRKs have also been predicted to regulate plant immunity. The SnRK1 subfamily members SnRK1 $\alpha / \beta / \gamma$ have been linked to resistance against diverse pathogens including virus, bacterium and fungus (Hulsmans et al. 2016). For example, the viral pathogenesis protein AL2/L2 interacts and inhibits the phosphorylation of SnRK1a for decreasing immune response, thus causing enhanced susceptibility of plant in Nicotiana benthamiana (Hao et al. 2003). The rice OSK35, as a homology of SnRK1 $\beta$, is a key regulator involved in resistance against both fungal and bacterial pathogens (Kim et al. 2015). Expression of the phosphorylated OSK35 protein is able to enhance resistance against rice blast and bacterial leaf blight (Kim et al. 2015). Arabidopsis SnRK1 $\beta / \gamma$-subunits interact with AtHSPRO1 and AtHSPRO2 and regulate disease resistance against bacterial pathogens (Gissot et al. 2006; Murray et al. 2007). SnRK3/CIPK subfamily members are also involved in regulation of plant immunity. TaCIPK10 from wheat confers to enhanced strip rust resistance through interacted with and phosphorylated TaNH2, which is homologous to AtNPR3/4 (Liu et al. 2019). Arabidopsis CIPK6 functions as a negative regulator of immunity against the bacterial pathogen (Sardar et al. 2017). In rice, OsCIPK14/15 likely play crucial role in the PAMP induced defense signaling pathway (Kurusu et al. 2010).

In the SnRK2 subfamily, two Arabidopsis SnRK2s, SnRK2.6/OST1 and SnRK2.8, participate in plant immunity (Melotto et al. 2006; Lee et al. 2015). OST1 functions together with signals such as nitric oxide (NO) and salicylic acid (SA), and has a pivotal role in response to biotic stress (Melotto et al. 2006). SnRK2.8 regulates plant immunity through the key immune regulator NPR1. SnRK2.8 can interact with and phosphorylate NPR1 to promote nuclear import of NPR1 from the cytoplasm (Lee et al. 2015). In rice, some of SnRK2s are involved in plant immunity. These SnRK2s include SAPK3, 5, 7 and 9, as their transcription levels could be induced by bacterial pathogen Xanthomonas oryzae pv. oryzicola (Xoc) infection (Xu et al. 2013). SAPK9 is able to form a protein complex with the molecular chaperones, OsSGT1 and OsHsp90, to regulate rice resistance to bacterial blight (Zhang et al. 2019). SAPK10 is also involved in bacterial pathogen resistance through phosphorylation WRKY72 to releases its suppression on jasmonic acid (JA) biosynthesis (Hou et al. 2019). However, the role of rice SnRK2s members in fungal pathogen resistance remains largely unknown. 
In this study, we report the identification of SAPK5 and its regulation on immunity in rice. SAPK5 posseses kinase activity and the 123th aspartic acid residue is essential for its kinase activity. Loss function of SAPK5 leads to compromised immune response and decreased resistance against Magnaporthe oryzae (M.oryzae) in rice. Furthermore, the phosphoryaltion of SAPK5 is involved in chitin induced immune response. Our study reveals that the SnRK2 family member SAPK5 positively regulates immunity and blast resistance in rice.

\section{Results}

\section{SAPK5 protein mainly distributes in cytoplasm}

It is well-known that the SnRK2 family proteins are involved in abiotic stress response (Kulik et al. 2011; Mao et al. 2019). However, the investigation of their roles in biotic stress is limited (Mao et al. 2019). In rice, the transcription levels of SAPK5, a member of SnRK2 family, can be induced by pathogen infection (Xu et al. 2013), suggesting that SAPK5 might be a regulator involved in rice immunity. However, the molecular characteristics of SAPK5 and its roles in rice immune response and disease resistance are still remain to be elucidated.

To characterize SAPK5, we firstly introduced a plasmid containing a gene expression cassette (35S.: GFPSAPK5) into rice protoplasts by PEG mediated transformation method to determinate the subcellular distribution of SAPK5 protein. As results shown in figure 1, green fluorescence signals can be mainly detected in cytoplasm in the cells expressing GFP-SAPK5 protein, whereas they were ubiquitously detected in the cells expressing GFP protein. These results indicate that SAPK5 is a cytoplasmic protein.

\section{The $123^{\text {th }}$ aspartic acid residue is essential for SAPK5 kinase activity}

SnRK2 members are suggested to be protein kinases which mainly relies on their phosphorylation (Kulik et al. 2011; Shinozawa et al. 2019). To ensure the protein kinase activity of SAPK5, we firstly expressed and purified the GST and GST-SAPK5 proteins from E.coli strain BL21 respectively and carried out an in vitro kinase activity assay. We found that a specific band was detected by anti phos-tag antibody, suggesting that GST-SAPK5 is a protein kinase possessing the autophosphorylation ability (Fig. 2). Then, we analyzed SAPK5 protein with PROSITE (https://prosite.expasy.org/prosite.html) and identified a predicated kinase active site, the $123^{\text {th }}$ aspartic acid residue (D), located in the predicted kinase domain. We then artificially replaced the $123^{\text {th }} \mathrm{D}$ of SAPK5 with leucine acid residue $(\mathrm{N})$ and purified the GSTSAPK5 ${ }^{\mathrm{D} 123 \mathrm{~N}}$ protein. When performed the similar kinase assay on GST-SAPK5 ${ }^{\mathrm{D} 123 \mathrm{~N}}$, no bands were detected by anti phos-tag antibody (Fig. 2). These results suggest that SAPK5 is a functional protein kinase. Moreover, the newly identified aspartic acid residue in the kinase domain is a novel residue that essential for its kinase activity.

\section{Disruption of SAPK5 compromise rice basal immune response}


To further investigate the genetic function of SAPK5 in rice immune response, we generated SAPK5 loss of function mutants, named as SAPK5KO, using CRISPR/CAS9 technology (Fig.3). The SAPK5KO plants exhibited significantly reduced tiller number, while other agronomic traits, including plant height, spikelet number per panicle, thousand seed weight, primary branch number per panicle and secondary branch number per panicle, were not obviously affected (Table 1 ).

Two genes, the OsKS4 and OsNAC4, are used as markers to evaluate the downstream immune response (Zhang et al. 2015). We thus examined the transcriptional expression levels of these two genes in SAPK5KO plants, in which SAPK5 is knocked out through CRISPR-based editing, post inoculation with the PAMP chitin. In wild type plants, the transcriptional levels of OsKS4 and OsNAC4 were induced about 100and 200-folds after chitin treatment, in comparison with the control (Fig. 4). However, the increased folds of these two genes in SAPK5KO plants were almost less than half of those in wild type plants (Fig. 4), indicating that immune response was inhibited in the plants with SAPK5 knocked out. These results suggest that SAPK5 is required for full immunity of rice plants and loss function of SAPK5 compromises rice basal immune response against.

\section{SAPK5 positively regulates blast resistance in rice}

Since our results showed that knock out of SAPK5 compromises chitin induced immune response in rice plants, we predicted that $S A P K 5$ may regulate fungal pathogen resistance of rice. To verify our hypotheses, we inoculated the SAPK5KO transgenic and wild type plants with M. oryzae. Four-week-old SAPK5KO plants and the wild type were challenged with blast isolate Zhong10-8-14 by punch-inoculation method. The lesion length and fungi biomass for each sample were measured and compared 7 days post inoculation (dpi). In accordance with the dampened immune response, SAPK5KO plants presented longer lesions and accumulated more fungi DNA than the control (Fig. 5), indicating that loss function of SAPK5 largely reduced rice resistance to the blast fungi. Our results clearly showed that $S A P K 5$ is indispensable for normal immune response and fungal pathogen resistance for rice.

\section{Chitin treatment elevates the phosphorylation level of SAPK5}

Since SAPK5 can be phosphorylated in vitro (Fig. 2), we speculated that phosphorylation of SAPK5 might also be associated with its mediated immune response and fungal pathogen resistance. Thus, we intend to test the basal immune response of SAPK5 at post-translational level. The Myc-SAPK5 transgenic rice plants were used and the phosphorylation level of SAPK5 upon chitin treatment was detected at $0,5,15$, 30,60 and 120 min respectively by western blot using anti phos-tag antibody. The result indicated that SAPK5 was quickly phosphorylated within 5 min post chitin treatment and the phosphorylation status continued at the remaining time points (Fig. 6). This result suggest that, in addition to transcription level, SAPK5 is also very likely regulate rice immunity through a post-translational mechanism.

\section{Discussion}

SAPK5 is an active protein kinase and the 123th aspartic acid residue is essential for its kinase activity 
Plant SnRK family consists of three subfamilies, SnRK1, 2 and 3 (Kulik et al. 2011). All the members of this family are protein kinases that can phosphorylate their substrates to regulate various biological processes (Kulik et al. 2011; Yu et al. 2014; Martínez-Barajas and Coello 2020). For example, plant protein kinase SnRK1 phosphorylates AL2 proteins from three subgroups of virus, resulting in a delay in viral DNA accumulation and symptom appearance (Shen et al. 2014). Arabidopsis SnRK2 subfamily members SnRK2.5, 2.6 and 2.10 interact with and phosphorylate the mRNA decapping complex member VARICOSE, controlling root development and response to salt (Kawa et al. 2020). The SnRK3 member CIPK23 interacts with and phosphorylates the voltage-gated inward $\mathrm{K}^{+}$channel (AKT1) required for $\mathrm{K}^{+}$acquisition in Arabidopsis to regulate low $\mathrm{K}^{+}$response (Li et al. 2006). In rice, in-gel kinase assay with MBP as substrate have revealed that all SnRK2 members, SAPK1 through 10, possess kinase activity (Kobayashi et al. 2004). However, their conserved kinase activity site(s) have not been identified. Interestingly, the 123th aspartic acid residue is responsible for the kinase activity of SAPK5 (Fig. 2), not only demonstrating that SAPK5 is an active protein kinase but also suggesting that in other SnRK2 members, the conserved residues corresponding to the 123th aspartic acid residue of SAPK5 are essential for their kinase activities.

\section{SAPK5 is essential for rice to fight against M. oryzae}

Emerging researches have revealed that some of SnRK2 proteins function in plant immunity regulation. In Arabidopsis, SnRK2.6/OST1 and SnRK2.8 confer to bacterial pathogen resistance (Melotto et al. 2006; Lee et al. 2015). Studies also have suggested that the rice SnRKs, including SAPK3, 5, 7, 9 and 10, are likely involved in resistance regulation against bacterial pathogen (Xu et al. 2013; Hou et al. 2019; Zhang et al. 2019). However, it is unknown whether SnRKs regulate resistance against fungal pathogens. Our study reveals that rice plant in knocking out of SAPK5 showed compromised immune response compared with the wild type plants (Fig. 4). Moreover, when challenged with the fungal pathogen M. oryzae, the rice plants with SAPK5 knocked out are more susceptible than the wild type (Fig. 5). Thus, SAPK5 is a novel immunity regulator which is essential for rice to fight against fungal pathogen $M$. oryzae.

\section{Phosphorylation cascades mediated by SAPK5 is likely required for plant immune response}

Protein phosphorylation is an important event as a post-transcriptional modification way that involved in plant immunity (Park et al. 2012). Since phosphorylation of a certain substrate is usually executed by protein kinases, thus the proper activation of protein kinases is pivotal for plant immunity. In plant, the mitogen-activated protein kinase (MAPK) cascades pathway is well know as it's a critical signaling module for immunity (Meng and Zhang 2013). For example, OsMAPKKKع/OsMAPKKK18 are phosphorylated and activated by OsRLCK185 upon chitin perception in rice (Wang et al. 2017; Yamada et al. 2017). The activated OsMAPKKKE/OsMAPKKK18 then phosphorylate OsMKK4/5, and OsMAPK3/6 are subsequently phosphorylated by OsMKK4/5 thus to burst immune response (Wang et al. 2017; Yamada et al. 2017). Previous studies also found that the SnRK2 family protein SnRK2.8 modulate plant immunity through phosphorylation of NPR1 to facilitate its nuclear importation from cytoplasm (Lee et al. 2015). Our study reveals that SAPK5 is able to be phosphorylated upon chitin treatment within 5 min 
(Fig. 6). The phosphorylation status of SAPK5 sustained at least 120 min (Fig. 6) or likely even more longer thus to guarantee the sufficient immune response. Although there is no evidence to indicate that NPR1 or other PR proteins are the directly targets of SAPK5 at this time, our present results also suggest that the phosphorylation signaling mediated by SAPK5 may play important roles in regulation of rice immunity and the phosphorylation of SAPK5 is likely a key switcher for immunity regulation.

\section{Conclusions}

We characterized a rice SnRK2 family member, SAPK5, and elucidated its molecular function in immune response and disease resistance. The SAPK5 protein is an active cytoplasmic kinase and the 123th aspartic acid residue is essential for its kinase activity. Loss function of SAPK5 attenuates chitin induced immune response and presents increased susceptibility to the fungal pathogen $M$. oryzae. We also found that the phosphorylation of the kinase SAPK5 is promoted upon chitin perception quickly, suggesting that phosphorylation play an important switch role for SAPK5 to function in plant immunity. Our study not only proves that SAPK5 is a novel key factor regulating plant immune response and disease resistance, but also provides new insights on understanding the machinery of SnRK2 family members on plant immunity.

\section{Methods}

\section{Plant materials and growth conditions}

For major agronomic traits analysis, the wild type Teipei309 (TP309) and the SAPK5KO plants were cultivated with three rows (ten plants per row) in the transgenic field at Sichuan Agricultural University in Wenjiang with three repeats. At maturity stage, four plants in the middle row were selected for agronomic traits analysis. Student's $t$ test was used to compare the differences between WT and SAPK5KO plants and the data were analyzed by Microsoft Excel 2019.

For evaluation of immune response and disease resistance, rice plants were grown in transgenic field for four weeks and then the leaves were harvested and used.

For protoplasts preparation, rice seedlings were grown in $1 / 2$ Murashige and Skoog (MS) medium in SoLo cup and incubated in growth chamber for about ten days before being used.

\section{Vectors construction and rice genetic transformation}

For construction of plasmids used in the study, the in vitro recombination method was employed by using the ClonExpress MultiS One Step Cloning Kit (P505-d1, Vazyme Biotech, China). The vectors and primers used were listed in Table S1.

CRISPR/CAS9-mediated gene knockout and rice genetic transformation were performed by Hangzhou Biogle Co., Ltd (Hangzhou, China) following stardard protocols. 


\section{Subcellular localization}

For subcellular localization analysis, a plasmid expression GFP-SAPK5 fusion protein was transformed into protoplasts prepared from Nipponbare seedlings following the method described previously (Bart et al. 2006). The protoplasts expression GFP protein alone were used as control. Fluorescence was examined under a laser confocal microscopy (NiKon A1 i90, LSCM, Japan) $18 \mathrm{~h}$ after transformation.

\section{RNA isolation and RT-qPCR/qPCR}

Total RNA was extracted using TRIzol agent (15596026, Invitrogen, USA) following the procedures described by the manufacturer. The RNA was subject to reverse transcription to synthesize first-strand cDNA by the PrimeScript ${ }^{\text {TM }}$ RT reagent Kit with gDNA Eraser (TRR047B, Takara, China) for removal of genomic DNA.

The reverse transcription-quantitative PCR (RT-qPCR) was conducted on a Bio-Rad CFX96 Real-Time System coupled to a C1000 Thermal Cycler (Bio-Rad, Hercules, USA). The reference gene UBIQUITIN 5 (UBQ5) (Zhu et al. 2016) was used as a reference. For DNA based qPCR, the primers specifically targeting genomic DNA were designed for M. oryzae MoPOT2 and rice UBIQUITIN 5 (OsUBQ5) genes (Li et al. 2017). Primer sequences are listed in Table S1.

\section{Protein expression, purification and in vitro phosphorylation assay}

The plasmids expression GST, GST-SAPK5 and GST-SAPK5 ${ }^{\mathrm{D} 123 \mathrm{~N}}$ were seperately transformed into a E.coli strain BL21 for protein purification. Bacteria were grown in Luria-Bertani (LB) medium containing $100 \mu \mathrm{g} / \mathrm{ml}$ Ampicillin at $37^{\circ} \mathrm{C}$ to $\mathrm{OD}_{600}=0.6$. After then, the bacterial cultures were supplemented with isopropyl $\beta$-D-1-thiogalactopyranoside (IPTG) to a final concentration of $1 \mathrm{mM}$ and incubated at $28^{\circ} \mathrm{C}$ for 8 hours for induction of heterogeneous protein expression. Protein purification, immunoblot analysis and in vitro autophosphorylation of the recombinant proteins were performed as described previously (Zhou et al. 2018). For detection of GST and the fusion proteins, anti-GST antibody was used (ab9106, Abcam, China) and phosphorylation status was detected by western blot with Phos-tag antibody (BTL-104, FUJIFILM, Japan).

\section{Chitin treatment and phosphorylation detection}

Leaf strips of 4-week-old Myc-SAPK5 transgenic rice plant was treated with $20 \mu \mathrm{g} / \mathrm{mL}$ chitin. Samples were respectively collected at $0,5,15,30,60$ and 120 min after treatment and subjected to phosphorylation detection by western blot with Phos-tag antibody (BTL-104, FUJIFILM, Japan). Expression level of Myc-SAPK5 protein was probed by anti-Myc antibody (ab9106, Abcam, China).

\section{Determination of rice immune response induced by chitin}

Leaf strips of 4-week-old SAPK5KO plants were treated with chitin $(20 \mu \mathrm{g} / \mathrm{mL})$ for $2 \mathrm{~h}$ as described before (Zhou et al. 2016). The expression levels of two PR genes, OsKS4 and OsNAC4, were determined by RT- 
qPCR and normalized to UBQ5 expression level. Water treatment was used as a control.

\section{Rice blast resistance assay}

Rice blast fungal punch inoculation assay was performed as described previously (Li et al. 2017). The lesion length was measured $7 \mathrm{dpi}$ and the relative fungal biomass was measured by genomic DNA based qPCR, primers used were listed in Table S1.

\section{Abbreviations}

SNF1: Sucrose non-fermenting 1; SnRK2:SNF1 related protein kinase 2; SAPK5:Osmotic stress activated protein kinases 5; PAMP:Pathogen-associated molecular pattern; AMPK:AMP activated protein kinase; $\mathrm{CBL}$ :Calcineurin B-like proteins; CIPK:CBL-interacting protein kinases; $\mathrm{ABA}$ :Abscisic acid; CRISPR:Clustered regularly interspaced short palindromic repeats; dpi:Days post inoculation; MAPK:Mitogen-activated protein kinase.

\section{Declarations}

\section{Authors' Contributions}

JW and $X Z$ conceived and designed research. $X L, W M, X Z, H S, H Y, W M$ and $L X$ performed experiments. JY, MH, WL, LS, LW, JL, HQ, YB, ML, KH, TQ, QH and XC provided other helps. JW and XZ analyzed data. $\mathrm{XZ}, \mathrm{JW}, \mathrm{XC}$ and $\mathrm{MH}$ wrote the manuscript. All authors read and approved the manuscript.

\section{Funding}

This study was supported by the National Natural Science Foundation of China (Grant No. 31922066 , 31701779 and 31825022) and the Science and Technology Department from Sichuan Province (Grant No. 2020JDJQ0040 and 2019YJ0432).

\section{Availability of Data and Materials}

All data supporting the conclusions of this article are provided within the article (and its additional files).

\section{Ethics Approval and Consent to Participate}

Not applicable.

\section{Consent for Publication}

Not applicable.

\section{Competing Interests}

The authors declare that they have no competing interests. 


\section{References}

Bart R, Chern M, Park CJ, Bartley L, Ronald PC (2006) A novel system for gene silencing using siRNAs in rice leaf and stem-derived protoplasts. Plant methods 2: 13

Gissot L, Polge C, Jossier M, Girin T, Bouly J-P, Kreis M, Thomas M (2006) AKIN ßy contributes to SnRK1 heterotrimeric complexes and interacts with two proteins implicated in plant pathogen resistance through its KIS/GBD sequence. Plant Physiology 142: 931-944

Hao L, Wang H, Sunter G, Bisaro DM (2003) Geminivirus AL2 and L2 proteins interact with and inactivate SNF1 Kinase. The Plant Cell 15: 1034-1048

Hou Y, Wang Y, Tang L, Tong X, Wang L, Liu L, Huang S, Zhang J (2019) SAPK10-mediated phosphorylation on WRKY72 releases its suppression on jasmonic acid biosynthesis and bacterial blight resistance. iScience 16: 499-510

Hrabak EM, Chan CWM, Gribskov M, Harper JF, Choi JH, Halford N, Kudla J, Luan S, Nimmo HG, Sussman MR, Thomas M, Walker-Simmons K, Zhu J-K, Harmon AC (2003) The Arabidopsis CDPK-SnRK superfamily of protein kinases. Plant Physiology 132: 666-680

Hulsmans S, Rodriguez M, De Coninck B, Rolland F (2016) The SnRK1 energy sensor in plant biotic interactions. Trends in Plant Science 21: 648-661

Kawa D, Meyer AJ, Dekker HL, Abd-El-Haliem AM, Gevaert K, Van De Slijke E, Maszkowska J, Bucholc M, Dobrowolska G, De Jaeger G, Schuurink RC, Haring MA, Testerink C (2020) SnRK2 protein kinases and mRNA decapping machinery control root development and response to salt. Plant Physiology 182: 361377

Kim C-Y, Vo KTX, An G, Jeon J-S (2015) A rice sucrose non-fermenting-1 related protein kinase 1, OSK35, plays an important role in fungal and bacterial disease resistance. Journal of the Korean Society for Applied Biological Chemistry 58: 669-675

Kobayashi Y, Yamamoto S, Minami H, Kagaya Y, Hattori T (2004) Differential activation of the rice sucrose nonfermenting1-related protein kinase 2 family by hyperosmotic stress and abscisic acid. The Plant Cell 16: 1163-1177

Kulik A, Wawer I, KrzywiDska E, Bucholc M, Dobrowolska G, yna (2011) SnRK2 protein kinases-key regulators of plant response to abiotic stresses. Omics : a journal of integrative biology 15 12: 859-872

Kurusu T, Hamada J, Nokajima H, Kitagawa Y, Kiyoduka M, Takahashi A, Hanamata S, Ohno R, Hayashi T, Okada K, Koga J, Hirochika H, Yamane H, Kuchitsu K (2010) Regulation of microbe-associated molecular pattern-induced hypersensitive cell death, phytoalexin production, and defense gene expression by calcineurin B-like protein-interacting protein kinases, OsCIPK14/15, in rice cultured cells. Plant Physiology 153: $678-692$ 
Lee H-J, Park Y-J, Seo PJ, Kim J-H, Sim H-J, Kim S-G, Park C-M (2015) Systemic immunity requires SnRK2.8-nediated nuclear import of NPR1 in Arabidopsis. The Plant Cell 27: 3425-3438

Li L, Kim B-G, Cheong YH, Pandey GK, Luan S (2006) A Ca ${ }^{2+}$ signaling pathway regulates a K+ channel for low-K response in Arabidopsis. Proceedings of the National Academy of Sciences 103: 12625-12630

Li W, Zhu Z, Chern M, Yin J, Yang C, Ran L, Cheng M, He M, Wang K, Wang J, Zhou X, Zhu X, Chen Z, Wang J, Zhao W, Ma B, Qin P, Chen W, Wang Y, Liu J, Wang W, Wu X, Li P, Wang J, Zhu L, Li S, Chen X (2017) A natural allele of a transcription factor in rice confers broad-spectrum blast resistance. Cell 170: 114126.e115

Liu P, Guo J, Zhang R, Zhao J, Liu C, Qi T, Duan Y, Kang Z, Guo J (2019) TaCIPK10 interacts with and phosphorylates $\mathrm{TaNH} 2$ to activate wheat defense responses to stripe rust. Plant Biotechnol J 17: $956-968$

Mao X, Li Y, Rehman SU, Miao L, Zhang Y, Chen X, Yu C, Wang J, Li C, Jing R (2019) The sucrose nonfermenting 1-related protein kinase 2 (SnRK2) genes are multifaceted players in plant growth, development and response to environmental stimuli. Plant and Cell Physiology 61: 225-242

Martínez-Barajas E, Coello P (2020) Review: How do SnRK1 protein kinases truly work? Plant Science 291: 110330

Melotto M, Underwood W, Koczan J, Nomura K, He SY (2006) Plant stomata function in innate immunity against bacterial invasion. Cell 126: 969-980

Meng X, Zhang S (2013) MAPK cascades in plant disease resistance signaling. Annual Review of Phytopathology 51: 245-266

Murray SL, Ingle RA, Petersen LN, Denby KJ (2007) Basal resistance against Pseudomonas syringae in Arabidopsis involves WRKY53 and a protein with homology to a nematode resistance protein. Mol Plant Microbe Interact 20: 1431-1438

Park C-J, Caddell D, Ronald P (2012) Protein phosphorylation in plant immunity: insights into the regulation of pattern recognition receptor-mediated signaling. Frontiers in Plant Science 3: eCollection 2012

Sardar A, Nandi AK, Chattopadhyay D (2017) CBL-interacting protein kinase 6 negatively regulates immune response to Pseudomonas syringae in Arabidopsis. Journal of Experimental Botany 68: 35733584

Shen W, Dallas MB, Goshe MB, Hanley-Bowdoin L (2014) SnRK1 phosphorylation of AL2 delays Cabbage leaf curl virus infection in Arabidopsis. J Virol 88: 10598-10612

Shinozawa A, Otake R, Takezawa D, Umezawa T, Komatsu K, Tanaka K, Amagai A, Ishikawa S, Hara Y, Kamisugi Y, Cuming AC, Hori K, Ohta H, Takahashi F, Shinozaki K, Hayashi T, Taji T, Sakata Y (2019) 
SnRK2 protein kinases represent an ancient system in plants for adaptation to a terrestrial environment. Communications Biology 2: 30

Tan W, Zhang D, Zhou H, Zheng T, Yin Y, Lin H (2018) Transcription factor HAT1 is a substrate of SnRK2.3 kinase and negatively regulates ABA synthesis and signaling in Arabidopsis responding to drought. PLOS Genetics 14: e1007336

Tian S, Mao X, Zhang H, Chen S, Zhai C, Yang S, Jing R (2013) Cloning and characterization of TaSnRK2.3, a novel SnRK2 gene in common wheat. Journal of Experimental Botany 64: 2063-2080

Wang C, Wang G, Zhang C, Zhu P, Dai H, Yu N, He Z, Xu L, Wang E (2017) OsCERK1-mediated chitin perception and immune signaling requires receptor-like cytoplasmic kinase 185 to activate an MAPK cascade in rice. Molecular Plant 10: 619-633

Xu M-R, Huang L-Y, Zhang F, Zhu L-H, Zhou Y-L, Li Z-K (2013) Genome-wide phylogenetic analysis of stress-activated protein kinase genes in rice (OsSAPKs) and expression profiling in response to Xanthomonas oryzae pv. oryzicola infection. Plant Molecular Biology Reporter 31: 877-885

Yamada K, Yamaguchi K, Yoshimura S, Terauchi A, Kawasaki T (2017) Conservation of chitin-induced MAPK signaling pathways in rice and Arabidopsis. Plant and Cell Physiology 58: 993-1002

Yu Q, An L, Li W (2014) The CBL-CIPK network mediates different signaling pathways in plants. Plant Cell Reports 33: 203-214

Zhang D, Liu M, Tang M, Dong B, Wu D, Zhang Z, Zhou B (2015) Repression of microRNA biogenesis by silencing of OSDCL1 activates the basal resistance to Magnaporthe oryzae in rice. Plant Science 237: 2432

Zhang F, Zeng D, Huang L, Shi Y, Chen T, Zhang F, Zhou Y (2019) Stress-activated protein kinase OsSAPK9 regulates tolerance to salt stress and resistance to bacterial blight in rice. Rice 12: 80

Zhong R, Wang Y, Gai R, Xi D, Mao C, Ming F (2020) Rice SnRK protein kinase OsSAPK8 acts as a positive regulator in abiotic stress responses. Plant Science 292: 110373

Zhou X, Liao H, Chern M, Yin J, Chen Y, Wang J, Zhu X, Chen Z, Yuan C, Zhao W, Wang J, Li W, He M, Ma B, Wang J, Qin P, Chen W, Wang Y, Liu J, Qian Y, Wang W, Wu X, Li P, Zhu L, Li S, Ronald PC, Chen X (2018) Loss of function of a rice TPR-domain RNA-binding protein confers broad-spectrum disease resistance. Proceedings of the National Academy of Sciences 115: 3174-3179

Zhou X, Wang J, Peng C, Zhu X, Yin J, Li W, He M, Wang J, Chern M, Yuan C, Wu W, Ma W, Qin P, Ma B, Wu X, Li S, Ronald P, Chen X (2016) Four receptor-like cytoplasmic kinases regulate development and immunity in rice. Plant, Cell \& Environment 39: 1381-1392 
Zhu X, Yin J, Liang S, Liang R, Zhou X, Chen Z, Zhao W, Wang J, Li W, He M, Yuan C, Miyamoto K, Ma B, Wang J, Qin P, Chen W, Wang Y, Wang W, Wu X, Yamane H, Zhu L, Li S, Chen X (2016) The multivesicular bodies (MVBs)-localized AAA ATPase LRD6-6 inhibits immunity and cell death likely through regulating MVBs-mediated vesicular trafficking in rice. PLOS Genetics 12: e1006311

\section{Figures}

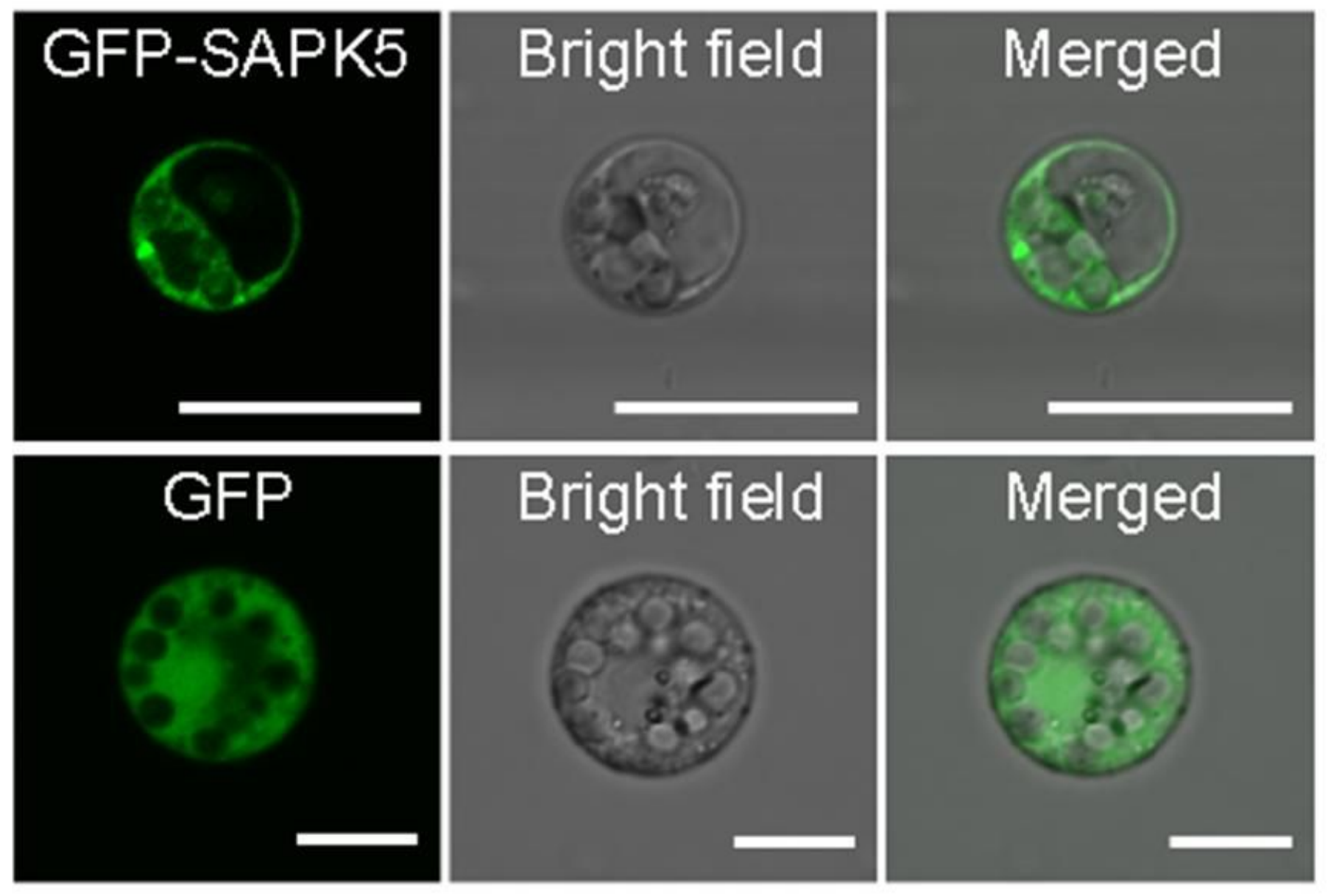

Figure 1

Subcellular localization of SAPK5 protein. The GFP-SAPK5 fusion protein was expressed in protoplasts prepared from Nipponbare. GFP protein alone was included as a control. Fluorescence was determined $18 \mathrm{~h}$ under laser confocal microscope post transformation. The green fluorescence represents GFP, Scale bars $=10 \mu \mathrm{m}$. 


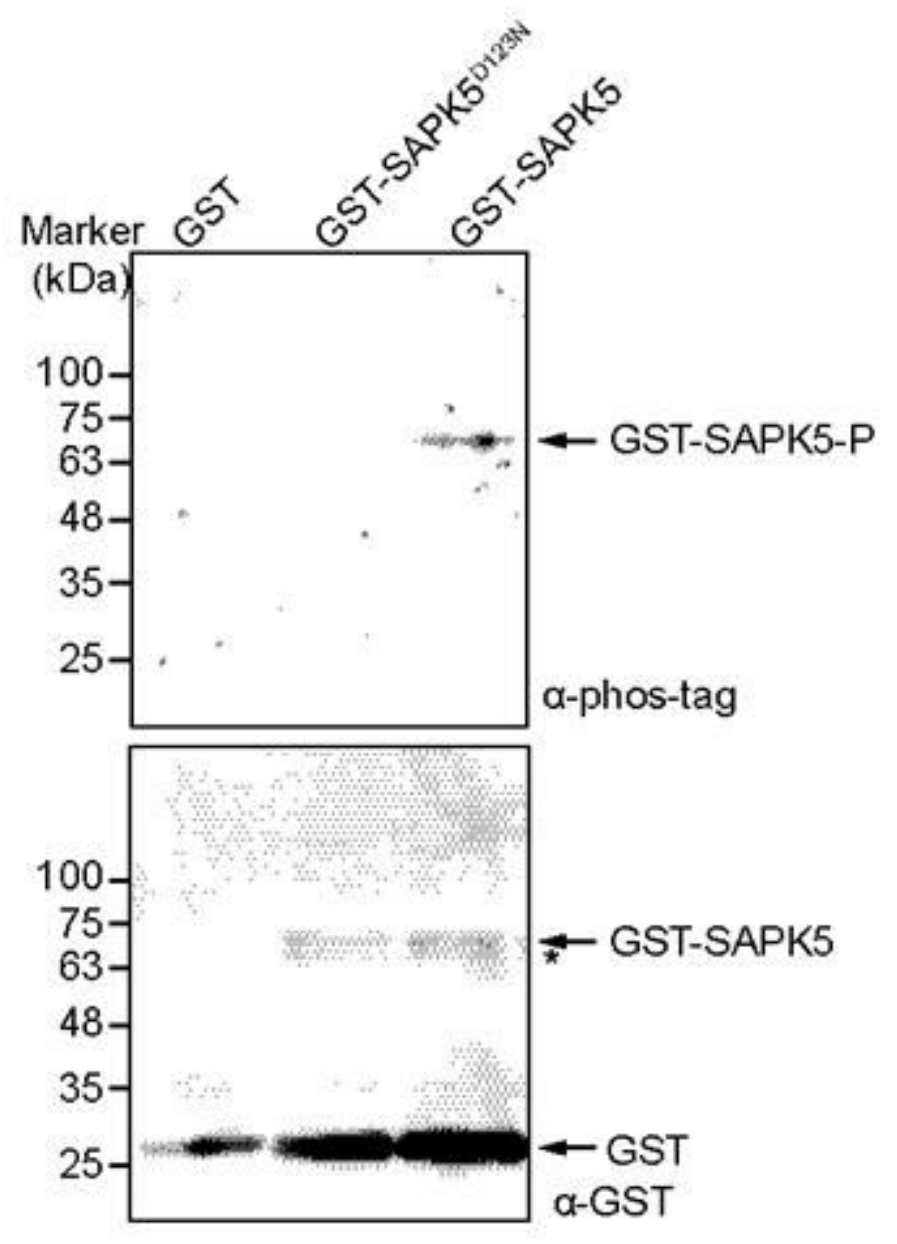

Figure 2

Determination of the kinase activity of SAPK5 protein in vitro. The recombinant proteins GSTSAPK5D123N, GST-SAPK5 and GST alone, were respectively expressed and purified from E. coli. The proteins were then incubated in kinase activity assay buffer. After incubation, proteins were subjected to western blot analysis with antibodies indicated. GST-SAPK5D123N is a putative kinase-dead mutant. The phos-tag antibody was used to detect phosphorylation of protein and the GST antibody was used to indicate the amount of each protein loaded. Asterisk indicates the nonspecific bands. 


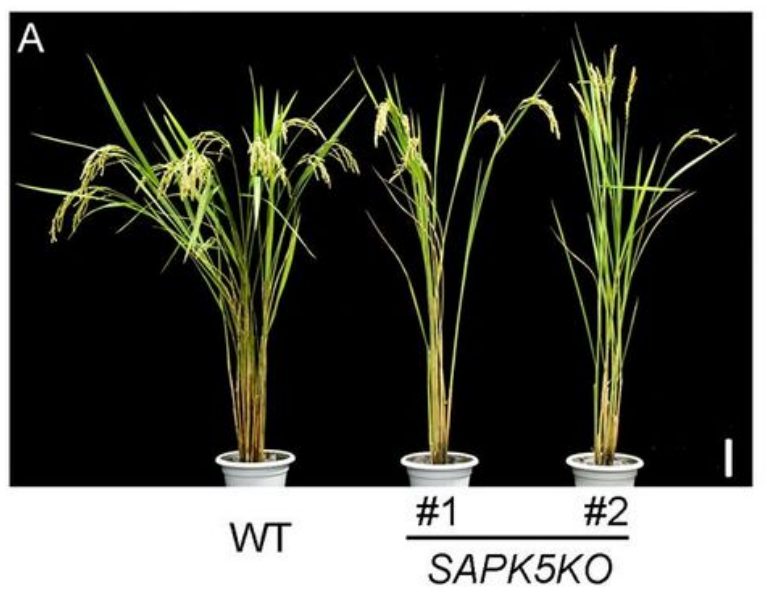

B

89

sgRNA

PAM 116

WT $\quad \cdots$ TGGCGATGAAGTTCATCGAGAGAGGGA $\cdots$

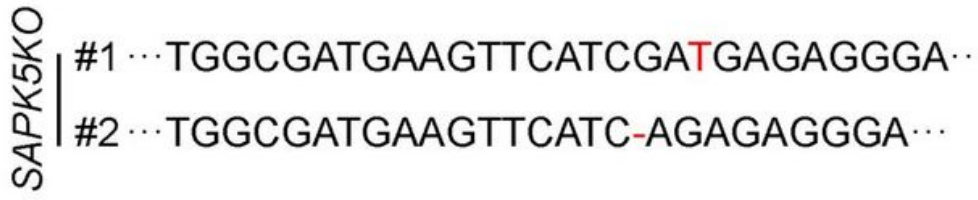

Figure 3

Generation of SAPK5 knockout rice plants by CRISPR/CAS9. (A) Photograph of two independent SAPK5 knockout (SAPK5KO) lines in rice TP309 (WT). (B) Sequence alignment between the WT and two SAPK5 knockout lines. A twenty nucleotides SAPK5 specific segment (indicated by wathet) was employed as sgRNA to achieve CRISPR/CAS9-mediated gene knockout. The PAM sequence was shown in blue and the mutations in SAPK5KO plants were presented in red.
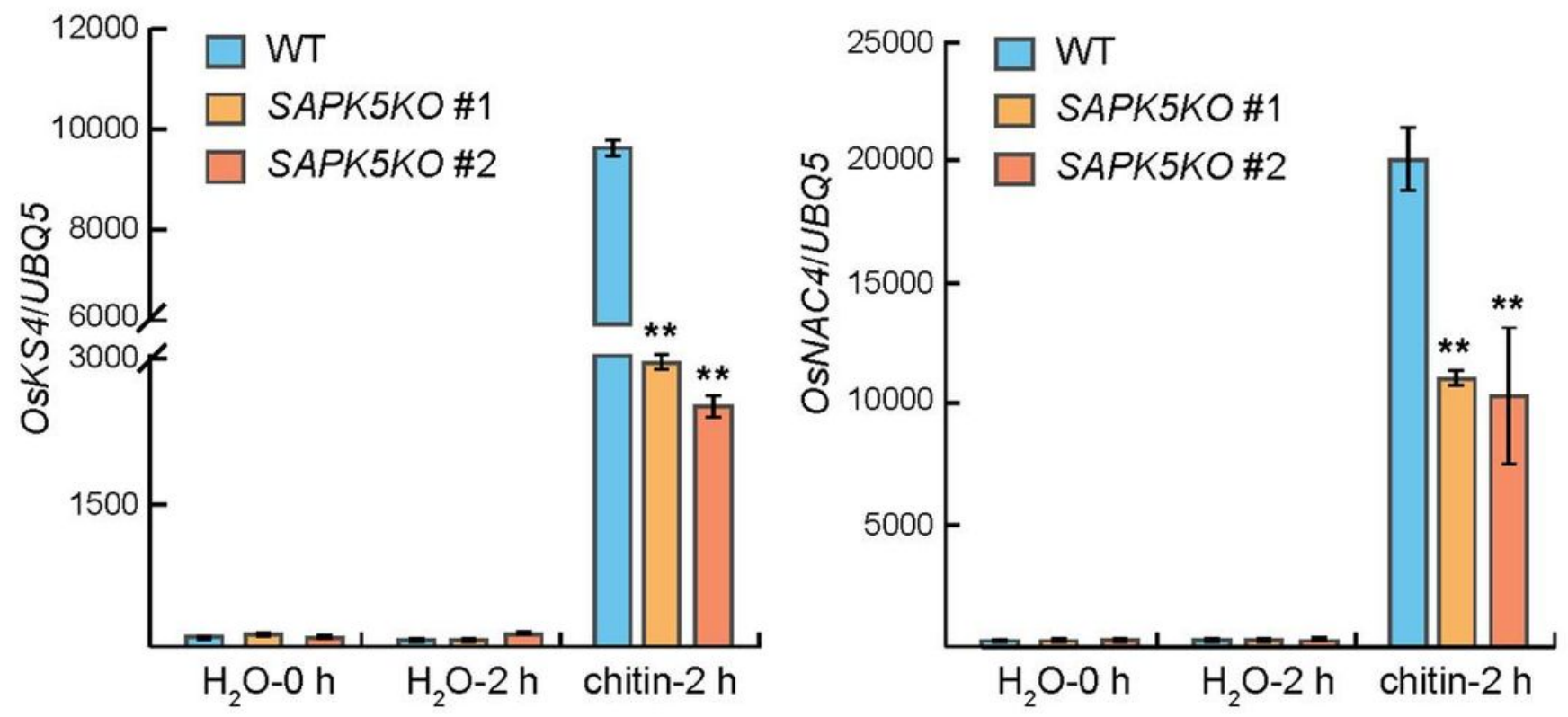

Figure 4 
Evaluation of chitin-triggered immune response in SAPK5KO rice plants. Leaf strips of 4-week-old plants were treated with $20 \mu \mathrm{g} / \mathrm{mL}$ chitin for $2 \mathrm{~h}$ and total RNA for each sample were extracted respectively. The expression levels of two PR genes, OsKS4 and OsNAC4, were determined by RT-qPCR and normalized to UBQ5 level. Water treatment was used as a control. This experiment was biological repeated three times with similar results. Data were obtained from three technical repeats (means $\pm S D$ ). Asterisks denote significant difference as determined by Student's t-test $(* \star, P<0.01)$.
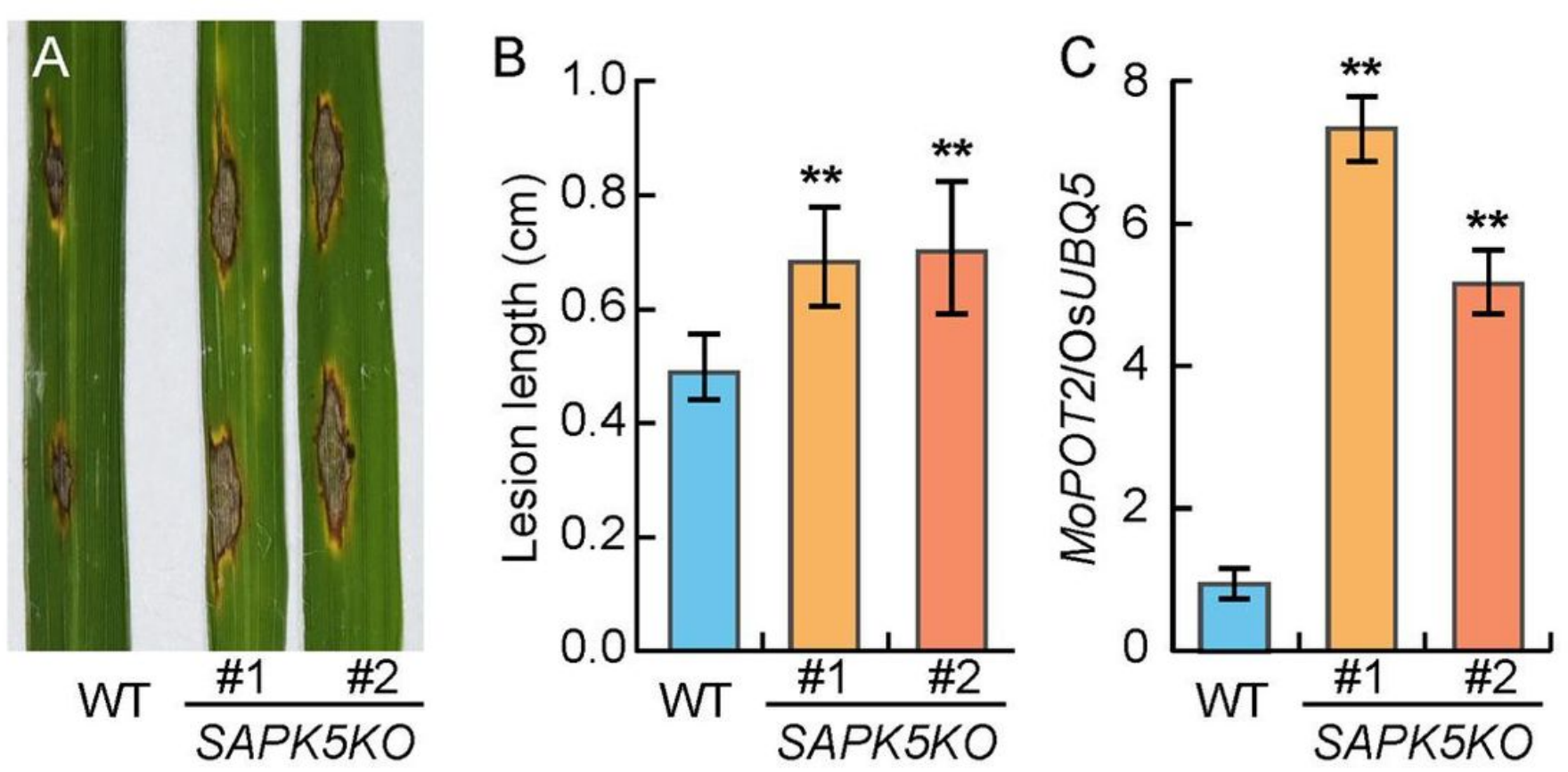

Figure 5

Determination on blast resistance of SAPK5KO rice plants. Photographic phenotype (A), lesion length (B) and relative fungal biomass $(C)$ of the two SAPK5KO rice plants inoculated with blast fungal isolate Zhong10-8-14 after 7 days. The relative fungi biomass was measured by genomic DNA based qPCR (MoPOT2/OsUBQ5). This experiment was biologically repeated for three times with similar results. Data were collected from three technical repeats (means $\pm S D$ ). Asterisks denote a significant difference as determined by Student's t-test $(* *, P<0.01)$. 


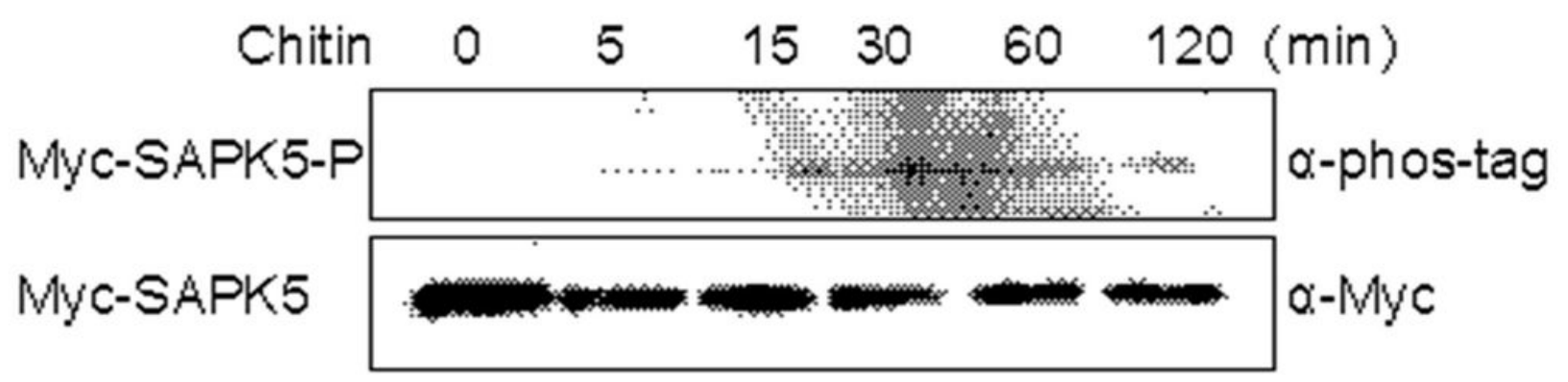

Figure 6

Analysis of SAPK5 phosphorylation status in response to chitin treatment in vivo. Leaf strips of 4-weekold Myc-SAPK5 transgenic rice were treated with $20 \mu \mathrm{g} / \mathrm{mL}$ chitin. Samples were collected at time points as indicated and subjected to phosphorylation detection by western blot with Phos-tag antibody. Expression level of Myc-SAPK5 protein was probed by anti-Myc antibody.

\section{Supplementary Files}

This is a list of supplementary files associated with this preprint. Click to download.

- TableS1.xlsx 